\title{
Spectrum engineering of multiwavelength erbium doped fiber lasers with intensity-dependent loss
}

\author{
Feng Li ${ }^{1 *}$ Xinhuan Feng, ${ }^{2,3}$ C. Lu, ${ }^{1}$ H. Y. Tam, ${ }^{2}$ P. K. A. Wai ${ }^{1}$ \\ ${ }^{1}$ Photonics Research Centre and Department of Electronic and Information Engineering \\ The Hong Kong Polytechnic University, Hung Hom, Kowloon, Hong Kong SAR, P. R. China \\ ${ }^{2}$ Photonics Research Centre, Department of Electrical Engineering, \\ The Hong Kong Polytechnic University, Hung Hom, Kowloon, Hong Kong SAR, P. R. China \\ ${ }^{3}$ Institute of Photonics Technology, Jinan University, Guangzhou, P. R. China \\ Presenting author e-mail address: *enlf@polyu.edu.hk
}

\begin{abstract}
We studied multiwavelength erbium-doped fiber lasers with intensity-dependent loss using rate equations. Pump power, cavity loss and the parameters of the intensity-dependent loss are varied to engineer the lasing spectrum. Experiments agree with simulation results.
\end{abstract}

\section{Introduction}

Multiwavelength fiber lasers are desirable in the applications of optical fiber communications, optical sensing, instrument testing, etc. The realization of multiwavelength $\mathrm{CW}$ lasers with erbium-doped fiber at room temperature by incorporating an intensity-dependent loss (IDL) element in the laser cavity have been shown in recent years [1-3]. In the proposed schemes, because of the complexity of the system, it is difficult to optimize the lasing spectrum by randomly varying the combination states of the multiple adjustable components in the cavity. By using an iterative laser model and a generic saturable homogeneous gain function, we showed that multiwavelength lasing is possible because the IDL can adaptively compensate for the gain at different wavelengths $[4,5]$. However, the simplicity of the gain model used also means that the results obtained in [4,5] are only qualitative and cannot capture the spectrum features which are important in experiments. In order to properly characterize the behaviors and to optimize the performance of multiwavelength erbium-doped fiber lasers, it is important to model the erbium gain accurately. In this paper, we study multiwavelength operation of erbium-doped fiber ring lasers by modeling the erbium-doped fiber gain using rate equations and real cross section parameters. We vary the pump power, cavity loss, and the parameters of the IDL to study the laser dynamics. Experimental studies on spectrum engineering agree qualitatively with the simulation results.

\section{Modeling and discussion}

Figure 1(a) shows the schematic diagram of a multiwavelength erbium doped fiber laser which is similar to the previous experimental schemes [1-3], which composes of a segment of erbium doped fiber (EDF) pumped at 1480 $\mathrm{nm}$ coupled from a WDM, an intensity-dependent loss, a comb filter to set the lasing channels, a variable optical attenuator (VOA) to adjust the constant cavity loss, an isolator (ISO) and an output coupler. In the simulations, the extra insertion loss induced by the optical elements and connection loss are all included in the loss of the VOA. The comb filter is assumed to be ideal which only set the wavelengths of the lasing channels. We further assume that the WDM can couple all pump power into the EDF, can couple all the residual pump power after a round trip out of the cavity, and would not attenuate the signals. The output coupler has a coupling ratio of $10 \%$ for all signals.

(a)

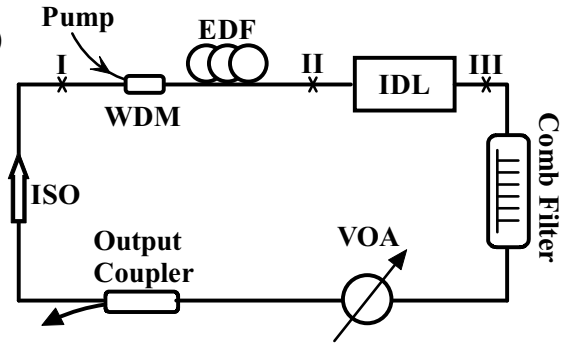

(b)

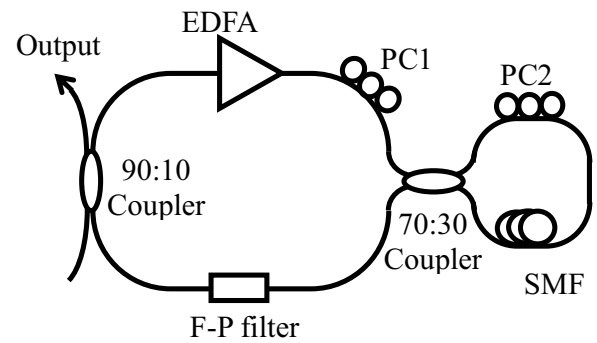

Fig. 1 (a) Schematic diagram and (b) experimental setup of a multiwavelength erbium-doped fiber ring laser.

Since we focused on the $\mathrm{CW}$ steady state solutions of the laser, we adopted the reduced rate equation model proposed in [6] to calculate the gain with multiwavelength input. The length of the EDF is assumed to be $15 \mathrm{~m}$ and the step size used in the simulations is $0.5 \mathrm{~m}$. The separation of the lasing channels is $0.8 \mathrm{~nm}$ which is defined by the comb filter. The transmission of the IDL based on nonlinear optical loop mirror or nonlinear polarization rotation 
can be described as

$$
T=\frac{T_{\max }+T_{\min }}{2}+\frac{T_{\max }-T_{\min }}{2} \cos [\phi+\varepsilon P \omega]
$$

where $T_{\max }$ and $T_{\min }$ are the maximum and minimum values of the transmission respectively, $\varepsilon$ is the nonlinearity of the IDL, $P$ is the incident power at angular frequency $\omega$, and $\phi$ is the phase shift of the cosine function.

We study multiwavelength laser operation of erbium-doped fiber ring lasers by varying the pump power, cavity loss, the phase shift and the minimum transmission of the IDL. The variation of maximum transmission and nonlinear coefficient of the IDL are considered equivalent to the adjustment of the loss of the VOA and pump power, so they are not varied in simulations. First we studied the effect of varying the pump power of the EDF. In these simulations, we assumed $T_{\max }=0.84, T_{\min }=0, \phi=0$ and $\varepsilon=1.38 \times 10^{-14} \mathrm{~W}^{-1} \mathrm{~s}$ for the IDL. The loss of the VOA is $2 \mathrm{~dB}$. We used a discrete laser model in which we iterate the effect of the erbium-doped fiber, IDL, VOA and output coupler successively [4,5]. Figure 2(a) shows the variation of lasing spectra with pump power. The color of the spectrum represents the power in $\mathrm{dBm}$ at different lasing channels. When the pump power equals $0 \mathrm{dBm}$, the laser cannot build up because the gain is lower than the cavity loss. As the pump power increases, the laser starts lasing and the number of lasing channels increases with the pump power. When the pump power exceeds $25 \mathrm{dBm}$, the lasing spectrum broadens rapidly especially on the long wavelength side. The asymmetry in the broadening spectrum is caused by the asymmetry of the EDF net gain profile at different upper level population density $n_{2}$. The lasing bandwidth at pump power of $35 \mathrm{dBm}$ is larger than $47 \mathrm{~nm}$. When the pump power is larger than $35 \mathrm{dBm}$, the laser becomes unstable and no steady state solution is obtained.
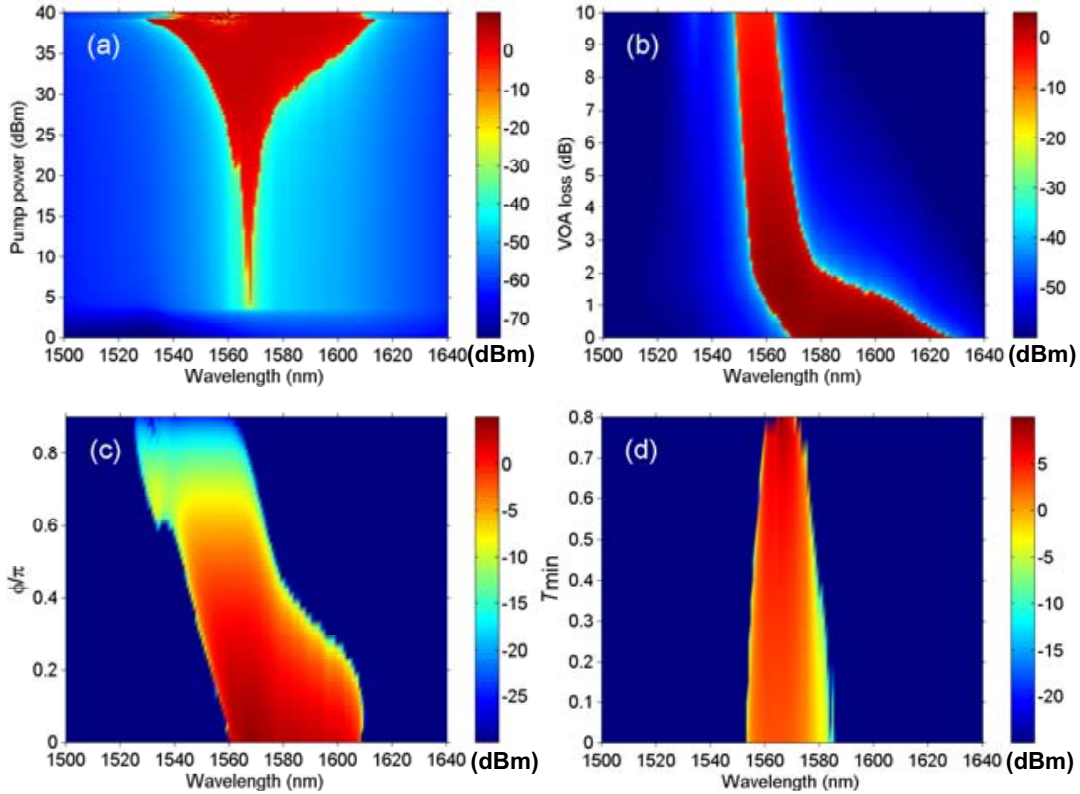

Fig. 2 Variations of the laser output spectra as a function of (a) pump power, (b) VOA loss, (c) phase offset, and (d) minimum transmission of the IDL. The colors indicate the lasing powers in $\mathrm{dBm}$.

We then studied the effect of cavity loss. We fixed the phase shift at 0 and the pump power at $30 \mathrm{dBm}$. Then we varied the VOA from 0 to $10 \mathrm{~dB}$. Figure 2(b) shows that when we increase the VOA loss, the spectrum shifts towards the shorter wavelength side and the bandwidth is also reduced, especially in the region $0-2 \mathrm{~dB}$. The lasing bandwidth is reduced because of the increase in lasing threshold which is predominantly controlled by the cavity loss. The spectrum shifts because when the cavity loss increases, the gain at steady state will have to increase in order to maintain gain loss balance. The upper level population density $n_{2}$ will therefore increase, which will shift the net gain profile $(g+\alpha) n_{2}-\alpha$ towards shorter wavelength [7].

The parameters of the IDL, phase shift, and minimum transmission will also affect the laser output. To study the effect of the phase shift parameter, we fixed the pump power at $30 \mathrm{dBm}$ and the VOA loss at $1 \mathrm{~dB}$. We varied the phase shift of the transmission curve of the IDL from 0 to $0.9 \pi$. When the phase shift is increased from zero, the initial value of the transmission curve is reduced, thus the lasing threshold is increased. But since the slope of the transmission is also increased, the spectrum is flattened which will increase the number of lasing channels. Because the two effects counteract, the bandwidth of the spectrum does not vary significantly as the phase shift increases but 
the power drops rapidly because of the increase in lasing threshold. When the phase shift approaches $\pi$, the IDL will work as a saturable absorber then multiwavelength operation is not possible [4,5]. Figure 2(d) shows the results when we fixed the phase shift at $0.1 \pi$ and varied the minimum transmission $T_{\min }$. When $T_{\min }$ increases from 0 to 0.5 , the lasing bandwidth decreases from 32 to $21 \mathrm{~nm}$ and the peak power increases from 2.8 to $5 \mathrm{dBm}$. Increase in $T_{\text {min }}$ decreases the slope of the transmission curve of the IDL, which weakens the compensation to the gain profile by the IDL. However, larger $T_{\min }$ will lead to smaller loss of the IDL which will increase the lasing power in the cavity. So we can obtain broader lasing bandwidth at the expense of weaker output power with smaller $T_{\min }$.

\section{Experiment}

To confirm our simulation results, we perform experiments with the setup shown in Fig. 1(b) which introduces periodic intensity-dependent loss by an NOLM. In [1], we have shown that the laser can operate in multiple wavelengths with uniform output power spectrum. Figure 3(a) shows the laser output power spectrum while we improve the pump power. The symbols are the peak powers of the output lasing channels. The wavelength spacing is $0.8 \mathrm{~nm}$ as determined by the F-P comb filter in the laser cavity. While the pump power increases, initially both the number of wavelengths and the output power of each wavelength increase. The whole spectrum will shift towards longer wavelength which is in agreement with Fig. 2(a).
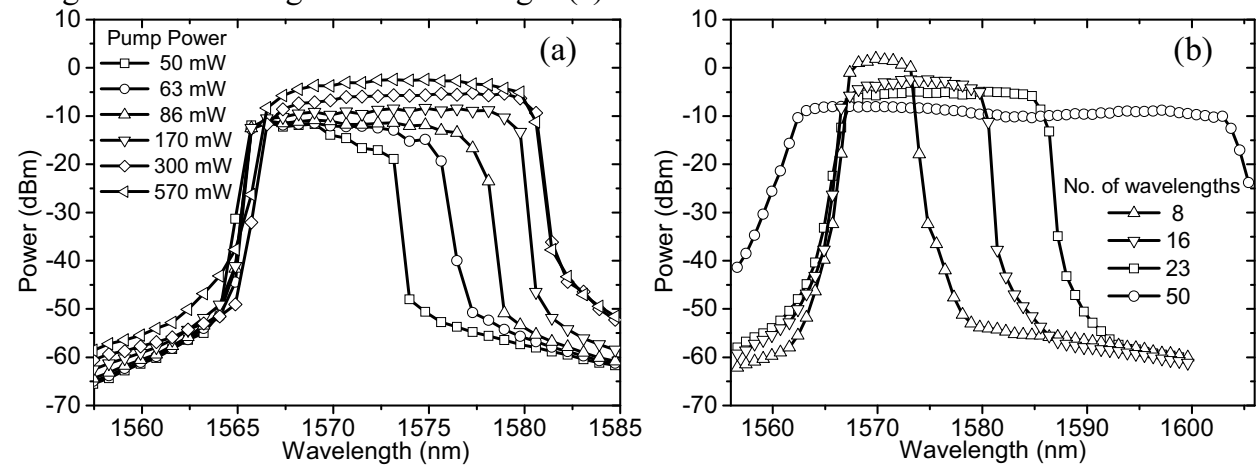

Fig. 3 Evolution of the output spectra of the laser for (a) different pump powers of the EDFA and (b) different settings of the polarization controller PC2.

To optimize the laser spectrum, the IDL should be adjusted by the PC in the cavity. Adjusting PC2 will change the transmission characteristics of the NOLM. Fig. 3(b) shows the spectrum variation when we adjust the polarization controller with fixed pump power. While PC2 is adjusted, the minimum transmission, phase shift and the constant loss of the cavity will be adjusted simultaneously, it is difficult to adjust the parameters individually in experiments. We observed that the spectrum can vary from outputting only 8 wavelengths at higher powers to 50 wavelengths at lower powers. The total power only varies by $2 \mathrm{~dB}$ while the peak power varies about $10 \mathrm{~dB}$ which agrees well with the simulation results with variation of $T_{\min }$. As the total power decreases about $2 \mathrm{~dB}$, the cavity loss and phase shift should have been adjusted too. While the number of wavelengths increases, the spectrum extends more towards the longer wavelength side instead of symmetrically. This can be easily obtained by the combination of adjusting cavity loss and phase shift, e.g. decrease the cavity loss and increase the phase shift simultaneously can extend the laser spectrum only on longer wavelength side.

\section{Summary}

We have characterized the operation of multiwavelength erbium doped fiber lasers with periodic IDL using a discrete laser model. Simulation results show that the lasing bandwidth will increase with the pump power, the lasing spectrum will shift towards the shorter wavelength when the cavity constant loss increases, and increasing the phase shift of the IDL will reduce the output power and flatten the spectrum. Increasing the minimum transmission will reduce the bandwidth but will enhance the laser output power. Experiments agree qualitatively with simulation results.

\section{References}

[1] X. Feng, H. Y. Tam, H. Liu, and P. K. A. Wai, Opt. Commun. 268, 278-281 (2006).

[2] X. Feng, H. Y. Tam, and P. K. A. Wai, Opt. Express 14, 8205-8210 (2006).

[3] X. Feng, P. K. A. Wai, C. Lu, H. Y. Tam, J. Li, and B. Guan, Optical Engineering 49, 074202 (2010)

[4] F. Li, H. Zheng, X. Feng, and P. K. A. Wai, in The 14th OptoElectronics and Communications Conference IEEE, Hong Kong, 2009 , TuG5.

[5] F. Li, X. Feng, H. Zheng, C. Lu, H. Y. Tam, J. N. Kutz, and P. K. A. Wai, Opt. Commun. 284, 2327-2336 (2011).

[6] C. R. Giles, and E. Desurvire, J. Lightwave Technol. 9, 271-283 (1991)

[7] E. Desurvire, Erbium-doped fiber amplifier: principles and applications, Wiley-Interscience, New York, 1994. 